S4 Table. Regression coefficients of the final clinical prediction models

\begin{tabular}{|c|c|c|c|c|c|}
\hline & & Coefficient & $\begin{array}{c}\text { Standard } \\
\text { error }\end{array}$ & Coefficient & $\begin{array}{c}\text { Standard } \\
\text { error }\end{array}$ \\
\hline \multirow{7}{*}{ Day and time of arrival } & & \multicolumn{2}{|c|}{ Clinical model } & \multicolumn{2}{|c|}{ Extended model } \\
\hline & Weekday evenings & .006 & .087 & .039 & .088 \\
\hline & Weekday nights & .201 & .113 & .233 & .114 \\
\hline & Weekend days & .180 & .093 & .193 & .094 \\
\hline & Weekend evenings & .057 & .121 & .092 & .122 \\
\hline & Weekend nights & .251 & .153 & .275 & .153 \\
\hline & Weekdays & \multicolumn{2}{|c|}{ reference } & \multicolumn{2}{|c|}{ reference } \\
\hline \multirow[t]{4}{*}{ Season } & Winter & .159 & .086 & .158 & .086 \\
\hline & Spring & .139 & .091 & .133 & .091 \\
\hline & Summer & -.012 & .095 & -.040 & .095 \\
\hline & Autumn & \multicolumn{2}{|c|}{ reference } & \multicolumn{2}{|c|}{ reference } \\
\hline \multirow[t]{5}{*}{ Age } & $<1$ years & .792 & .121 & .979 & .123 \\
\hline & $1-<2$ years & .307 & .131 & .490 & .133 \\
\hline & $2-<5$ years & .146 & .121 & .286 & .122 \\
\hline & $5-<12$ years & .006 & .118 & .075 & .118 \\
\hline & $12-16$ years & \multicolumn{2}{|c|}{ reference } & \multicolumn{2}{|c|}{ reference } \\
\hline \multirow{12}{*}{$\begin{array}{l}\text { Sex } \\
\text { Presenting problem }\end{array}$} & Female & -.067 & .065 & -.071 & .065 \\
\hline & Shortness of breath & .634 & .177 & .646 & .177 \\
\hline & ENT problems & -.185 & .229 & -.162 & .230 \\
\hline & Gastro-intestinal problems & .960 & .159 & .838 & .161 \\
\hline & Neurological problem & .840 & .200 & .789 & .200 \\
\hline & Unwell child & .571 & .161 & .493 & .162 \\
\hline & Urological problems & .231 & .273 & .070 & .275 \\
\hline & Rash & -.197 & .228 & -.115 & .229 \\
\hline & Abscess and soft tissue infection & .792 & .265 & .795 & .265 \\
\hline & Wounds & -.737 & .188 & -.848 & .193 \\
\hline & Trauma & -1.035 & .336 & -.896 & .336 \\
\hline & Other & \multicolumn{2}{|c|}{ reference } & \multicolumn{2}{|c|}{ reference } \\
\hline \multirow[t]{3}{*}{ Triage urgency } & Emergent / very urgent & .654 & .120 & .518 & .120 \\
\hline & urgent & .603 & .077 & .511 & .078 \\
\hline & Standard / non-urgent & \multicolumn{2}{|c|}{ reference } & \multicolumn{2}{|c|}{ reference } \\
\hline Tachycardia & present & .266 & .088 & .251 & .089 \\
\hline Tachypnoea & present & .169 & .088 & .165 & .088 \\
\hline Temperature & $>=38.0$ degrees Celsius & -.030 & .098 & -.089 & .099 \\
\hline \multirow{6}{*}{$\begin{array}{l}\text { Oxygen saturations } \\
\text { Level of consciousness } \\
\text { Laboratory tests } \\
\text { Imaging } \\
\text { IV medication or fluids } \\
\text { Intercept }\end{array}$} & Oxygen saturation $<94 \%$ & .406 & .233 & .355 & .233 \\
\hline & Reduced & .432 & .256 & .335 & .257 \\
\hline & Any & - & - & .454 & .079 \\
\hline & Any & - & - & .478 & .088 \\
\hline & Any & - & - & .637 & .110 \\
\hline & & -5.541 & & -5.869 & \\
\hline
\end{tabular}

Legend:

Clinical model:

Linear predictor $=-5.541+0.006 *$ Time of presentation: Weekday evenings $+0.201 *$ Time of presentation:

Weekday nights $+0.180 *$ Time of presentation: Weekend days $+0.057 *$ Time of presentation: Weekend 
evenings $+0.251 *$ Time of presentation: Weekend nights (reference:Time of presentation: Weekdays) +0.159 * Season: Winter $+0.139 *$ Season: Spring + -0.012* Season: Summer (reference: Season: Autumn) + $0.792 *$ Age: $<1$ years $+0.307 *$ Age: $1-<2$ years $+0.146 *$ Age: $2-<5$ years $+0.006 *$ Age: $5-<12$ years (reference: Age: $12-16$ years) $+-0.067 *$ Sex: Female $+0.634 *$ Presenting problem: Shortness of breath $+-0.185 *$ Presenting problem: ENT problems $+0.960 *$ Presenting problem: Gastro-intestinal problems $+0.840 *$ Presenting problem: Neurological problem $+0.571 *$ Presenting problem: Unwell child $+0.231 *$ Presenting problem: Urological problems $+-0.197 *$ Presenting problem: Rash $+0.792 *$ Presenting problem: Abscess and soft tissue infection $+-0.737 *$ Presenting problem: Wounds $+-1.035 *$ Presenting problem: Trauma (reference: Presenting problem: Other) $+0.654 *$ Triage urgency: Emergent / very urgent $+0.603 *$ Triage urgency: urgent (reference: Triage urgency: Standard / non-urgent) $+0.266 *$ Tachycardia present $+0.169 *$ Tachypnoea present $+-0.030 *$ Temperature $>=38.0$ degrees Celsius $+0.406 *$ Oxygen saturation $<94 \%+.432$

* Level of consciousness - Reduced

\section{Extended model:}

Linear predictor: $-5.869+0.039 *$ Time of arrival: Weekday evenings $+0.233 *$ Time of arrival: Weekday nights $+0.193 *$ Time of arrival: Weekend days $+0.092 *$ Time of arrival: Weekend evenings $+0.275 *$ Time of arrival: Weekend nights (reference: Time of arrival: Weekdays) $+0.158 *$ Season: Winter $+0.133 *$ Season: Spring + $0.040 *$ Season: Summer (reference: Season: Autumn) $+0.979 *$ Age: $<1$ years $+0.490 *$ Age: $1-<2$ years + $0.286 *$ Age: $2-<5$ years $+0.075 *$ Age: $5-<12$ years (reference: Age: $12-16$ years) $+-0.071 *$ Sex Female + $0.646 *$ Presenting problem: Shortness of breath $+-0.162 *$ Presenting problem: ENT problems $+0.838 *$ Presenting problem: Gastro-intestinal problems $+0.789 *$ Presenting problem: Neurological problem $+0.493 *$ Presenting problem: Unwell child $+0.070 *$ Presenting problem: Urological problems $+-0.115 *$ Presenting problem: Rash $+0.795 *$ Presenting problem: Abscess and soft tissue infection +-0.848 * Presenting problem: Wounds $+-0.896 *$ Presenting problem: Trauma (reference: Presenting problem: Other) $+0.518 *$ Triage urgency Emergent / very urgent $+0.511 *$ Triage urgency urgent (reference: Triage urgency Standard / nonurgent $)+0.251 *$ Tachycardia present $+0.165 *$ Tachypnoea present $+-0.089 *$ Temperature $>=38.0$ degrees Celsius $+0.355 *$ Oxygen saturation $<94 \%+0.335 *$ Level of consciousness - Reduced $+0.454 *$ Laboratory tests $+0.478 *$ Imaging $+0.637 *$ IV medication or fluids

To calculate risk prediction: $1 / 1+(\exp$ (-linear predictor))

$\mathrm{Cl}$ confidence interval; ENT Ear nose and throat; IV intravenous; 